Rueda Gascó, Paloma.

Profesora asociada Universitat de València. Departamento Didáctica de la expresión musical, plástica y corporal. CREARI Grupo de Investigación en Pedagogías Culturales GIUV2013-103.

\title{
¿Por qué no vamos a los colegios? Sobre la ausencia de arte actual en la escuela.
}

\section{¿Why don't we go to the schools? About the absence of the art nowadays in the school.}

\author{
TIPO DE TRABAJO:
}

Comunicación.

PALABRAS CLAVE:

Arte y educación, escuela, arte colaborativo, educación primaria, taller artista.

KEY WORDS:

Art and education, school, collaborative arts projects, primary education, artist studio.

\section{RESUMEN.}

Este texto ahonda en una de las conclusiones extraídas de una investigación anterior, que señalaba la necesidad de reivindicar la de acción de artistas (entre otros especialistas de diversos campos) en el contexto escolar. Sabemos de las ventajas del arte participativo; conocemos todas las posibilidades de los proyectos colaborativos de artistas en diversos contextos y con diferentes colectivos; muchos son los países que cuentan con programas con financiación estatal para la participación de artistas en colegios e institutos. Sin embargo el desarrollo de proyectos dinamizados por artistas en el contexto escolar, no pasan de ser contados casos aislados en nuestro país.

Vivimos actualmente un creciente interés del mundo del arte hacia la educación por sus posibilidades mediadoras y emancipadoras. Sin embargo, este interés se enfoca mayoritariamente hacia entornos no formales e informales. La hipótesis desde la que se propone este estudio es que existe un tradicional rechazo por parte de los profesionales de las artes visuales hacia el mundo de la escuela. Este texto se centra en esta idea, que será revisada y contrastada por medio de un pequeño estudio de casos.

\section{ABSTRACT.}

This text deepns one of the conclusions drawn from an earlier research, which pointed out the need to claim the action of artists (among other specialists from several areas) in the school context. We know the advantages of community art works. We know all the possibilities of the collaborative projects of artists in different contexts and with different groups; Many countries have state-funded programs for the participation of artists in Primary and Secondary schools. However, the development of projects dynamized by artists in the school context, are no more than isolated cases in our country.

We are currently experiencing an increasing interest in the art world towards education because of its possibilities about mediation and emancipation. However, this interest is mostly focused on non-formal and informal environments. The hypothesis from which this study is proposed is that there is a traditional rejection by professionals of the visual arts towards the world of the school. This text focuses on this idea, which will be reviewed and contrasted through a brief case study. 


\section{CONTENIDO.}

\section{Introducción:}

La proliferación de espacios, propuestas y proyectos imbricados entre la práctica artística y la educativa es actualmente una realidad: "El mundo del arte ha reclamado lo pedagógico como espacio que también le puede ser propio" (Sánchez De Serdio 2010, 45). Iniciativas como las Jornadas anuales QUAM del ACVic Centre d'Arts Contemporànies, el grupo Las Lindes (grupo de investigación y acción acerca de educación, arte y prácticas culturales ${ }^{1}$ ), el festival de Educación Expandida ZEMOS98, o prácticas de mediación cultural como Medialab Madrid, colectivo Núbol, plataforma Transductores, Pensart Mediación Cultural, etc. son reflejo de cómo "La dimensión educativa entra de lleno también en las prácticas artísticas en nuestros días, no como un elemento complementario o práctica secundaria, sino como una clave de la producción del conocimiento y del trabajo de las políticas culturales" (Rodrigo 2009, 249). Vidagañ (2016) recupera todo tipo de propuestas artísticas que indagan en torno a las conexiones entre arte y educación, como el proyecto Do it de Ulrich Obrist, Cabanyal archivo vivo, la exposición Un saber realmente útil en el Centro de Arte Reina Sofia o el trabajo El museo es una escuela de Luis Camnitzer.

Si bien es clave pensar en la escuela como un lugar para la experimentación y la implementación de metodologías provenientes del arte, la inclusión del arte contemporáneo en las aulas sigue siendo una tarea pendiente (excepto en ciertos casos puntuales) en la institución escolar: "La escuela primaria y secundaria olvidan el arte y, si lo valoran, excluyen el arte contemporáneo" (Calaf 2006, 23). Agra constataba esta realidad en 2001, denunciando la inexistencia de itinerarios de formación en arte, sobre todo arte moderno y contemporáneo, que eduquen en los modos de observar, hacer, hablar y conocer (Agra 2001). Finalizada la escolaridad, el concepto de arte que los jóvenes pueden haber construido gracias a su experiencia en la escuela, sigue teniendo muchos más elementos comunes con una concepción más propia de la modernidad que de propuestas actuales:

Si nos acercamos a la escuela bajo esta perspectiva, si entramos en sus aulas y observamos lo que cuelga de sus paredes, lo primero que observamos es que el arte contemporáneo se (con)funde con el arte moderno y que son los nombres de Paul Klee, Kandinsky, Picasso, Miró, los artistas que guían e inspiran, de manera destacada, las prácticas educativas, lo que no deja de ser una manifestación de un seguimiento de un cierto canon artístico, al tiempo que muestra el interés de la escuela por lo existente y no por lo emergente (Hernández 2013, 119).

Una de las más graves carencias de la educación formal para escolares de hasta doce años de edad (Educación Infantil y Primaria) actualmente en nuestro país, es la inexistencia de especialistas de las artes visuales en el contexto escolar. A los maestros de Educación Primaria se suman en los colegios los especialistas de Lengua Extranjera, Audición y Lenguaje, Pedagogía Terapéutica, Educación Física y Música. Sin embargo, ni rastro de especialistas en Artes Visuales. La educación artística para estudiantes de hasta doce años está en manos de las maestras y maestros de Infantil y Primaria. La mirada de estos profesionales hacia el mundo de las artes y la cultura visual es, en una penosa mayoría, limitadora, parcial, acrítica y superficial, como consecuencia de una insuficiente formación en este campo.

A pesar de los esfuerzos por revertir esta situación por parte del profesorado del área de artes visuales en las facultades de Magisterio, la formación en educación artística para maestros continúa arrastrando una serie de graves dificultades: mínima presencia en los estudios de Grado en Maestro de Infantil y de Primaria y escasez de horas (solo una asignatura cuatrimestral obligatoria relacionada con artes visuales). En definitiva, toda una trayectoria de marginalidad que todavía sigue impidiendo la existencia de un itinerario que forme a Especialistas en Artes (al mismo nivel que los ya existentes Especialistas de Música o Educación Física) para Educación Primaria. La educación artística para niños en estas condiciones, llevadas al extremo de la degradación con las leyes actuales (LOMCE y sus correspondientes Decretos autonómicos), perpetúan una generalidad de alarmante situación de la educación artística en el contexto escolar (generalidad que, como toda norma, es salvada por excepcionales y muy brillantes casos).

Paralelamente a todos los esfuerzos que actualmente tratan de visibilizar las artes y reivindicar su importancia en la educación obligatoria (Huerta y Dominguez 2016), se hace pertinente buscar nuevas soluciones acordes a las circunstancias actuales. Una de ellas es la colaboración de artistas profesionales en el desarrollo de proyectos artísticos en los colegios. Muchos son los países que incentivan programas de participación de artistas en sus escuelas:

En Nueva Zelanda, el programa Education's Artists in Schools, ofrecido por el Ministerio de Educación, facilita la inmersión de artistas profesionales (de la danza, el teatro, música y artes visuales) en las escuelas de Primaria y Secundaria (Hernández 2009), proporcionando a las escuelas el acceso a artistas que trabajan en las escuelas como artistas residentes o visitantes ${ }^{2}$.

\footnotetext{
${ }^{1} \mathrm{http}: / / \mathrm{ca} 2 \mathrm{~m}$. org/es/videoteca-lindes

${ }^{2}$ http://artsonline2.tki.org.nz/artistsined/profiles.php
} 
También con un modelo de artista residente Room13 se presenta como una red de estudios gestionados y dirigidos por escolares. El primer estudio original nació en Escocia en 1994 para ir poco a poco extendiéndose, incluyendo escuelas urbanas internas en Londres, Los Ángeles y Mumbai, espacios comunitarios en diferentes municipios de Sudáfrica, escuelas de aldeas rurales en la India o un orfanato en Nepal (Gibb 2013). Cada Room 13 consiste en un aula en un colegio (u otras instituciones como las anteriores) que se ofrece como taller para artistas en residencia. Evitando cualquier tipo de instrucción formal, los artistas residentes trabajan con las niñas y niños, no enseñándoles como un maestro sino invitándoles a trabajar con ellos como artistas, desafiándoles con una intención seria y promoviendo la discusión y la construcción de un discurso crítico desde un posicionamiento horizontal en el que tratar a los niños como artistas e intelectuales (recordemos su lema "A qué edad puedes empezar a ser artista?") ${ }^{3}$.

De nuevo en Inglaterra, el proyecto What is Artists in Schools? da respuesta a los objetivos del curriculum nacional en cuanto a la inmersión en las diversas formas de arte de los estudiantes desde edad temprana. Este programa facilita el contacto entre artistas y escuelas, proponiendo a éstas diversos proyectos (desde talleres de un día o de una semana como planes de residencia de artistas a largo plazo) diseñados y desarrollados por artistas en los colegios ${ }^{4}$.

Con ciertas similitudes en su formato, encontramos en nuestro contexto más cercano el programa Aquí trabaja un artista. Éste se planteó como un proyecto de residencias artísticas desarrolladas en instituciones educativas, promovido por el CA2M y realizado con el apoyo económico de la Fundación Daniel y Nina Carasso en Madrid: durante el curso 2014-2015, Pilar Álvarez y Alejandro Simón trabajaron como artistas residentes en dos colegios de Primaria seleccionados de entre la red Enterarte. Un seminario final sirvió de valoración coral del proyecto y la publicación Entre la escuela y el museo. Evaluación de acompañamiento de residencias artísticas en colegios de primaria Aquí trabaja un artista 2014-2015, expone detalladamente la experiencia (Álvarez 2016).

También en Madrid, desde 2014 y continuando en la actualidad, el proyecto Levadura se propone como un programa de residencias de creadores en escuelas. Levadura lanza cuatro modalidades de participación para artistas: creación de las artes visuales, cultura digital, arquitectura y relación con la ciudadanía y creación musical y sonora. Periódicamente se abren convocatorias de proyectos diseñados por diferentes artistas en cada uno de estos cuatro campos, que son propuestos a colegios de Infantil y Primaria para ser desarrollados conjuntamente. Es un proyecto co-producido por el Área de las Artes del Ayuntamiento de Madrid y la Fundación Banco Santander, que tiene lugar en Matadero Madrid, Centro Cibeles de Cultura y Ciudadanía, Medialab-Prado y Centro Cultural Conde Duque. Levadura surge de la colaboración entre Matadero Madrid y la Asociación Pedagogías Invisibles en el seno del Grupo de Educación Disruptiva, como parte del programa de pensamiento crítico que se lleva a cabo a lo largo de todo el año en Matadero Madrid $^{5}$.

Si bien países como Inglaterra cuentan con un potente respaldo institucional para promover la presencia de artistas en la escuela (dado que es un objetivo a lograr según el curriculum oficial para la educación primaria y secundaria y una acción promovida por la propia administración), nuestro contexto es más hostil respecto a este tipo de propuestas: por un lado, el marco legislativo no solo no acompaña sino que actualmente está enfocado a la extinción de las artes en la enseñanza reglada. Las estructuras institucionales y administrativas propias de cada campo (artes y educación) encuentran pocas conexiones, con lo que las dificultades organizativas y de financiación acaban con casi cualquier iniciativa de colaboración. Por otra parte, la formación de los profesionales de las artes visuales y la de los profesionales de la educación siguen recorridos completamente distintos promoviendo un perfil humano con pocos puntos en común. Sin embargo:

Si las instituciones del arte y las escuelas están dispuestas a explorar el territorio que las separa y a la vez las conecta (y las administraciones públicas a reconocer el valor de este tipo de propuestas y darles apoyo) es posible que se empiecen a abrir vías de transformación, más significativas precisamente porque se basan en el trabajo desde la diferencia y no desde lo mismo (Sánchez De Serdio 2010,58)

Efectivamente, es posible crear formas de colaboración que generen nuevos formatos de participación, nuevas relaciones entre artistas y estudiantes y nuevas formas de aprendizaje en torno a las artes, probablemente más significativas y transformadoras que muchas de las actuales. Sabidos los beneficios que pueden traer consigo los proyectos colaborativos de artistas en la escuela, la pregunta a plantear sería: ¿qué interés puede suscitar el desarrollo de proyectos de este tipo para los artistas y demás profesionales provenientes del mundo de las artes? ¿Qué factores han favorecido la existencia de proyectos en esta línea, en casos como los que hemos revisado? Y ¿cómo podemos llevar estas prácticas a otros contextos?

\footnotetext{
${ }^{3}$ http://room13international.org/

4 http://www.artistsinschools.co.uk/

${ }^{5}$ http://levadura.mataderomadrid.org/
} 


\section{Metodología:}

La hipótesis desde la que se propone este estudio es que existe un tradicional rechazo por parte de los profesionales de las artes visuales hacia el mundo de la escuela. Para analizar esta idea desde una metodología que combina análisis cualitativo y cuantitativo, diseñamos un cuestionario que pregunta sobre diferentes factores presentes en las escuelas y que pueden ser posibles generadores de estas reacciones negativas respecto a la institución escolar. Para ello, recogemos la voz de profesionales de las artes, arquitectura y diseño que no se dediquen exclusivamente a la producción artística sino que tengan también cierto interés, vocación o acercamiento hacia la educación. Así, los participantes en el estudio son las alumnas y alumnos del Máster de Secundaria, en su especialidad de Dibujo de la Universitat de València en el curso 2016-2017 (en su mayoría licenciados en Bellas Artes, también un amplio grupo de arquitectos y con algunos casos de diseñadores y restauradores).

El estudio está enmarcado en una investigación más amplia en torno a la relación entre arte contemporáneo y entornos educativos, que se desarrolla con una metodología mixta de investigación. En ella se proponen diversas estrategias experimentales en las diferentes fases del estudio, por ejemplo el análisis de creaciones artísticas realizadas específicamente para la investigación por parte de profesionales de las artes visuales en torno a la temática planteada. Éstas se combinan con herramientas clásicas propias de la investigación cualitativa, cuantitativa y artística (entrevistas, análisis de imágenes, narrativa autobiográfica, etc.) con el objetivo central de revisar las posibilidades de acercamiento entre entorno escolar y artistas profesionales, valorar posibles interferencias que dificultan este diálogo, y visibilizar las ventajas que podría suponer una relación más fluida arte-escuela.

\section{Primeros resultados:}

Con un $61,5 \%$ del total, los factores estéticos de los colegios (estética general, estética pasillos y estética aulas) emergen como los elementos menos atractivos o incluso desagradables para los participantes. Si a estos estímulos visuales sumamos el $19 \%$ que suponen los olores y sonidos de los colegios, nos encontramos con que un $79 \%$ del total de las respuestas tiene que ver con elementos sensoriales de los colegios: estética, olores y sonidos.
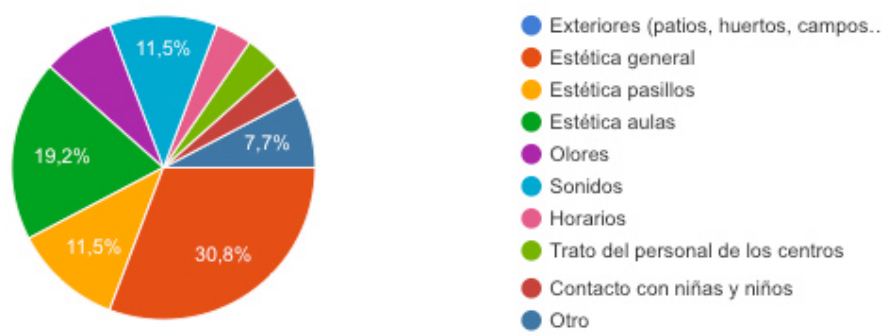

Fig.1.- Gráfico de las respuestas a la pregunta "En general, ¿sabrías decir qué elementos propios de los colegios te resultan menos atractivos, más incómodos o desagradables?"

Pensando en las aulas de los colegios, los artistas participantes señalan el mobiliario y la distribución del espacio como elementos negativos y la luz como un factor altamente positivo (seguido de las imágenes en las paredes).
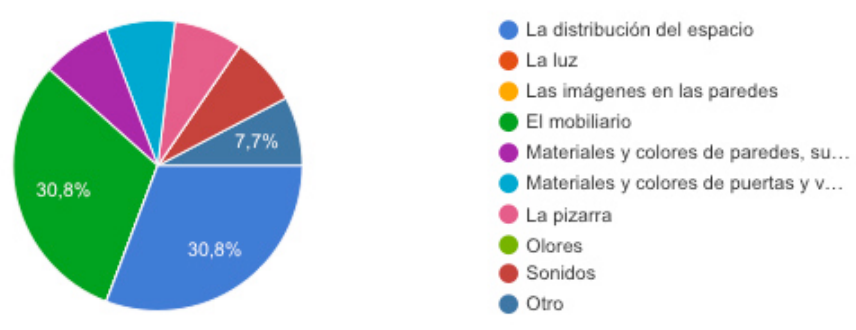

Fig.2.- Gráfico de las respuestas a la pregunta “Centrándonos en las aulas, ¿qué factores recuerdas como menos agradables?”. 

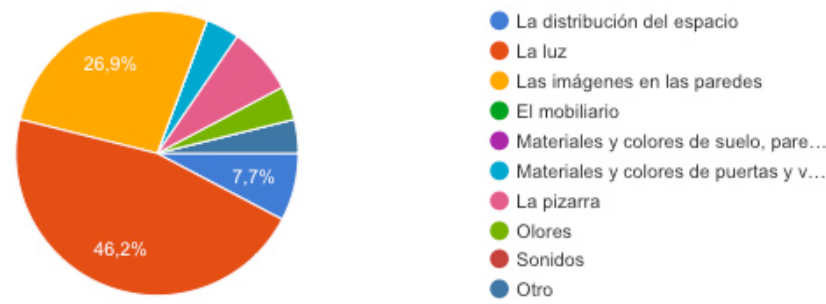

Fig.3.- Gráfico de las respuestas a la pregunta “Centrándonos en las aulas, ¿Qué factores recuerdas como más agradables?”.

Efectivamente, en nuestro colectivo de artistas participantes los factores estéticos en particular y estímulos sensoriales en general, actúan como fuente de atracción o rechazo hacia los colegios. Es posible que el recuerdo de los estímulos sensoriales sea potenciado cuando nuestra memoria recupera experiencias significativamente intensas como son las vividas en la escuela. Y es posible que estos estímulos estén directamente vinculados con rechazos o atracciones que en la actualidad generamos de manera inconsciente individualmente. Sea como sea, los estímulos sensoriales se presentan como un factor clave en nuestra relación con los entornos educativos (Rueda 2015). Cuestión diferente son los rechazos construidos y aprendidos social y culturalmente, por ejemplo los reproducidos aún en las facultades de Bellas Artes en torno a una consideración de la acción docente como una opción personal y profesional menor o de bajo interés.

El colectivo participante en este pequeño estudio parece haber superado dichos prejuicios tal y como demuestran un llamativo $100 \%$ de respuestas afirmativas a la pregunta: "¿ ¿Te interesaría trabajar como artista colaborador/a con colegios de Infantil y Primaria como opción profesional?” y el $96 \%$ de respuestas afirmativas a la pregunta “¿Te interesaría ser artista residente en un colegio? Esto supondría recibir un salario y disponer de un aula de un colegio como estudio para tu trabajo personal (en arte, diseño, arquitectura, etc.) y recibir a grupos de niños para trabajar contigo a ciertas horas (como forma de aprendizaje en educación artística)". Más allá de pensar en esta opción como salida profesional, las respuestas mayoritarias destacan los posibles beneficios personales de experiencias como éstas, con adjetivos como "gratificante, enriquecedor, divertido, interesante", etc.

\section{Conclusiones:}

Valorada positivamente la opción de proponer acciones en la línea de los casos revisados en nuestra introducción y dada la buena acogida (en el contexto de este breve estudio) de la posibilidad de trabajar en colegios de Primaria por parte del colectivo de artistas participante, pensamos en los pasos siguientes a dar en cuanto a la temática planteada. Podemos deducir dos necesidades principales a las que atender en el camino hacia una implantación más extendida de programas que vinculen a profesionales de las artes con la educación Infantil y Primaria en el entorno escolar: por una parte, promover la reflexión, y un conocimiento en profundidad desde el cuestionamiento de estereotipos y prejuicios por parte de los profesionales al respecto de las instituciones implicadas (tanto por parte de los artistas hacia la institución escolar, como desde los profesionales de la educación hacia el mundo del arte). En segundo lugar, analizar pormenorizadamente las diferencias respecto a las estructuras institucionales de los campos en juego: regulaciones, cuestiones administrativas, económicas y organizativas, con el fin de crear nuevas vías estables y mantenidas en el tiempo entre profesionales de las artes (asociaciones de artistas, centros de creación, galerías, museos, etc.) y de la educación (en el caso que nos ocupa, colegios de Educación Infantil y Primaria). Entendemos que la creación de redes de colaboración sostenidas y a largo plazo ha de pasar necesariamente por estas dos acciones previas.

\section{FUENTES REFERENCIALES.}

AGRA, M. J. 2001. Itinerarios de arte para la educación. Aula de Innovación Educativa [Versión electrónica], 106,pp. 1-3.

ÁLVAREZ, M. 2016. Entre la escuela y el museo. Evaluación de acompañamiento de residencias artísticas en colegios de primaria Aquí trabaja un artista 2014-2015. Madrid: Oficina de Cultura y Turismo.

CALAF, R. 2006. Cruzar miradas. En R. CALAF y O. FONTAL (coords.) Miradas al patrimonio. Gijón: Trea.

HERNÁNDEZ, F. 2013. Experiències de relació dels infants i els joves amb l'art contemporani a l'escola i al museu. En Arts, educació $i$ interdisciplinarietat. Els proyectes, punt de trobada entre museus i escola. Tarragona: Museu D’Art Modern de la Diputació de Tarragona. 

http://dx.doi.org/10.4995/ANIAV.2017.5817

HERNÁNDEZ, F. 2009.¿De qué hablan los artistas cuando realizan proyectos artístico-pedagógicos? En Acciones reversibles. Arte, educación y territorio. Vic: ACVIC. Centre D’Arts Contemporànies. Eumo Editorial.

HUERTA, R. y DOMINGUEZ, R. 2016. Las reivindicaciones del profesorado de Educación Artística ante los nuevos obstáculos curriculares; el proyecto "Second Round: Art i Lluita a Secundària". EARI Educación Artística Revista de Investigación, 7, pp.10-18.

GIBB, C. 2012. Room 13: The Movement and International Network. International Journal of Art and Design Education, 31(3), pp.237244.

RODRIGO, J. 2009. El trabajo en red y las pedagogías colectivas: retos para una producción cultural. En Acciones reversibles. Arte, educación y territorio. Vic: ACVIC. Centre d’Arts Contemporànies. Eumo Editorial.

RUEDA, P. 2015. Entornos de Educación Artística no formal: estudio de casos de academias de arte privadas en Valencia. Tesis Doctoral: Universitat de València.

SANCHEZ DE SERDIO, A. 2010. Arte y educación: diálogos y antagonismos. Revista Iberoamericana de Educación, 52, pp.43-60.

VIDAGAÑ, M. (2016). Museos, mediación cultural y artes visuales: perfiles profesionales del ámbito educativo. Tesis Doctoral: Universitat de València. 